\title{
View-Factor Approach as a Radiation Model for the Reentry Flowfield
}

\author{
Daniil A. Andrienko* \\ University of Michigan, Ann Arbor, Michigan 48109 \\ Sergey Surzhikov \\ Institute for Problems in Mechanics, 119526, Moscow, Russia \\ and \\ Joseph Shang \\ Wright State University, Dayton, Ohio 45435 \\ DOI: $10.2514 / 1 . A 33305$
}

\begin{abstract}
An accurate and efficient radiation transfer model, based on the view-factor approach, is proposed for the reentry flowfield. The radiation transfer equation is coupled to the gasdynamic system of equations in order to describe the energy transfer in the absorbing and emitting air plasma in two-dimensional axisymmetric and three-dimensional geometries. A newly derived semi-analytical expression for the radiative flux density dramatically simplifies the calculation of the spectral and integral characteristics of the radiation flowfield in the axisymmetric geometry. The comparison with the ray-tracing method and tangent slab approximation revealed an asymptotic accuracy of radiation flux density.
\end{abstract}

\section{Nomenclature}

$A=$ surface of element

$F, E=$ incomplete elliptic integral of first and second kind

$J=$ spectral intensity of radiation

$J_{b}=$ spectral intensity of the black body

$T=$ attenuation factor

$V \quad=$ volume of element

$W \quad=$ radiation flux density

$\kappa_{\nu}=$ absorption coefficient

$\tau \quad=$ optical thickness

Subscripts

$i, j \quad=\quad$ indices of gasdynamic grid

$\nu \quad=$ spectral index

\section{Introduction}

$\mathbf{S}$ UPERORBITAL reentry experiments [1-3] have revealed a significant contribution of radiation transfer into the total heat flux on the surface of a space vehicle. At the same time, a large number of studies that deal with the problem of an accurate and efficient simulation of radiation transfer have been published over the past decades. However, the problem of radiation transfer in the nonequilibrium gas, heated by a shock wave, is not yet completely solved. One of the possible reasons for this is a strong dependence of radiation properties from chemical composition and thermodynamic state of high-temperature gas. Another factor that complicates the numerical simulation of radiation transfer is the mathematical complexity of the radiation energy conservation equation. The integral radiation intensity depends on the spatial and angular coordinates as

Presented as Paper 2014-2488 at the 45th AIAA Plasmadynamics and Lasers Conference, AIAA Aviation and Aeronautics Forum and Exposition 2014, Atlanta, GA, 16-20 June 2014; received 19 March 2015; revision received 6 August 2015; accepted for publication 11 September 2015; published online 25 November 2015. Copyright (C) 2015 by the American Institute of Aeronautics and Astronautics, Inc. All rights reserved. Copies of this paper may be made for personal or internal use, on condition that the copier pay the $\$ 10.00$ per-copy fee to the Copyright Clearance Center, Inc., 222 Rosewood Drive, Danvers, MA 01923; include the code 1533-6794/15 and $\$ 10.00$ in correspondence with the CCC.

*Postdoctoral Research Fellow, Department of Aerospace Engineering, 1320 Beal Ave.

†Professor, Director, pr. Vernadskogo 101-1.

*Research Professor, 3640 Col Glenn Hwy. well as on the wavelength. These problems make the numerical simulation of radiation transfer very prohibitive in multidimensional geometries.

An efficient and accurate model of radiation transfer is currently a very desirable item in aerothermodynamics. Until recently, the tangent slab (TS) approximation [4] was generally used to evaluate the radiation flux incident on the heat shield of a space vehicle, while the radiation heating of the leeward surface was often neglected due to its relatively small contribution and the inaccuracy of the TS approximation for nonshock flows. With increasing computational resources, the ray-tracing method (RTM) became available [5]. One of the important advantages of the RTM is the accurate description of radiation flux for the entire flowfield [6,7]. However, the RTM is computationally expensive, especially in multidimensional geometries. Another disadvantage of the RTM is a necessary approximation of the computed flowfield on the tracing ray, involving an algorithm of the nearest neighbor search (NNS). This approximation is required even when no estimation of optical thickness of the tracing ray is required. While a significant effort must be spent to obtain a high-quality, artifact-free flowfield that captures a shock wave []], inaccurate approximation may compromise the overall accuracy of the radiation transfer model.

On the other hand, the spherical harmonics method [9] offers a relatively simple and efficient way to account for the multidimensional radiation transfer utilizing the same mesh, used for the solution of gasdynamic equations. Recently, the $P_{1}$ approximation was successfully applied to develop a two- and three-dimensional model of radiation transfer for the entry of a Martian probe $[10,11]$. While the reasonable agreement with the RTM was achieved, the $P_{1}$ approximation was found to give inaccurate results in the optically transparent gas.

Among other radiation transfer models, the view-factor (VF) approach can be used to access the heat flux on the surface of a space vehicle. This approach, also known as the zonal method, was originally proposed in [12] to account for the radiation energy exchange between isothermal elements separated by an absorbing and emitting media. The key idea of the VF approach is to establish the parameter of visibility between the elements participating in the radiative energy exchange. In the case of nonparticipating media, the visibility parameter simply equals to the fraction of the solid angle at which the emitting element is observed by the receiving element. Hence, the visibility parameter is often referred to as a VF. When the media between exchanging elements has its own radiative properties, the attenuation of radiation is often included in the definition of the VF. The latter can be established for the surface-to-surface, 
volume-to-surface, and volume-to-volume types of energy exchange [13], regarding the particular geometry. This paper concentrates on the second of three cases, which takes place when the surface of a space vehicle is heated by the shock flowfield.

The VF approach is an efficient tool to determine the radiation flux or temperature in the multidimensional radiating enclosure. The indisputable advantage of this method is the possibility to derive the $\mathrm{VF}$ in the closed form for some geometric configurations. A comprehensive review of some analytical formulas is given in [14]. One should note that the VF approach generates an asymptotically accurate solution; hence, this method should be considered as one of the few that can be applied in the wide range of input parameters and demonstrate high efficiency and accuracy.

However, the VF approach has attained little attention in aerothermodynamics. The use of the VF approach under the assumption of optically transparent media between the shock layer and the surface of a space vehicle is performed in $[15,16]$. Radiation heating rates were obtained for the Titan Aeroshell $\overline{\mathrm{NASA}}$ mission [17]. It is shown that the VF approach is generally more accurate than the TS approximation. The latter severely overestimates the radiation heating rate at the shoulder of the heat shield. Improvement of the accuracy is contrasted to the increased cost of the radiation transfer model by a factor of two orders of magnitude. The main reason for this is that no attempt was made to obtain an expression for the VF in a closed form.

Meanwhile, efficiency is a crucial aspect of a radiation transfer model. The state-of-the-art calculations of spectral and integral characteristics of radiation field in a shock-heated gas at temperatures of a few tens of thousands degrees involve millions of integration points on the wavelength scale. These calculations are often referred to as the line-by-line optical model [18]. Taking into account that the integration over the angular and spatial coordinates is also required, the computational efficiency of the radiation transfer model becomes a vital element. However, in some cases, there is no need for an excessive accuracy of the spectral model, because the nonequilibrium model of chemically reactive gas may also contain a large uncertainty [19]. An alternative approach, used in the present paper, is to divide the spectral range into a number of intervals of nonuniform length and perform an averaging of optical properties within each interval. Previously, this approach has been successfully applied to resolve spectral radiation heating in a number of space missions [10,20,21].

The present paper extends the VF approach to the problem of radiation transfer during the flight in Earth atmosphere. The VF approach is investigated in the air plasma, generated in the vicinity of the RAM-C II reentry probe [22]. This choice of a flight experiment is due to the verified applicability of the multitemperature model to the reentry flow at moderate velocities [23]. The radiation flowfield is treated from the two- and three-dimensional points of view, although the reentry of the probe occurred with the zero angle of attack. This is done to access the accuracy and efficiency of the VF approach in multidimensional geometries.

An important novelty of the present research is the closed analytical relations for the spectral radiation flux density on the surface of space vehicle in the axisymmetric geometry. Although it can be done only assuming an optical transparent gas, these computational relaxations demonstrate an asymptotically accurate and extremely efficient way of estimation of the radiation flux. This method can be incorporated in the spectral model of any complexity and used for the estimation of radiation flowfield at high altitudes, when the absorption of radiation is generally insignificant. The VF approach is compared with the ray-tracing and TS methods. The radiation transfer model is applied to the flow at different altitudes, from nearly transparent to strongly absorbent flows.

In the present paper, the VF approach is coupled to the NavierStokes solver. For the description of thermally and chemically nonequilibrium flowfield, the standard finite rate chemistry module and equations of trans-rotational, vibrational, and electron-electronic energy conservation are adopted. The three-temperature model of chemically reactive, viscous, conductive, and radiative hypersonic flow is applied to the wide range of altitudes. The present paper concentrates on the improving of accuracy and efficiency of existing radiation transfer model, rather than on the validation against available flight data. This is due to the complexity and large uncertainty of the multiphysical models, presently adopted in aerothermodynamics. However, it was shown before that the thermodynamic, spectral, and radiation transfer models, considered in the present paper, are capable of describing the experimental data [24].

The structure of the paper is as follows. The governing equations of radiation transfer are described in Sec. II. The nonequilibrium physical and chemical models are briefly reviewed in Sec. III. The test case and the derivation of final relations for two-dimensional axisymmetric geometry are discussed in Sec. IV. Analysis of radiative heating rates using two- and three-dimensional relaxations is given in Sec. $\underline{\mathrm{V}}$, followed by conclusions.

\section{A. View-Factor Approach}

\section{Radiation Transfer Model}

The governing equations of radiation energy transfer are derived to describe the propagation of electromagnetic energy in nonscattering, emitting, and absorbing media under the assumption of local thermal equilibrium (LTE). The latter is important because it allows simplification of the source term in the radiation transfer equation when the Boltzmann population of energy levels is assumed. Deviation from equilibrium may occur in a strong shock wave. However, the LTE is widely used in a large number of engineering applications. While the VF approach can be coupled with the thermodynamic model to account for the nonequilibrium radiation [16], the focus of the present paper is the numerical simulation of radiation transfer.

The radiation transfer equation in nonscattering, emitting, and absorbing media under the assumption of LTE can be formulated for the spectral radiative intensity $J_{\nu}(s, \Omega)$ :

$$
\frac{\partial J_{\nu}(s, \Omega)}{\partial s}+\kappa_{\nu}(s) J_{\nu}(s, \Omega)=\kappa_{\nu}(s) J_{b, \nu}(s)
$$

where $s$ and $\Omega$ are the vectors of coordinates and solid angle, $\kappa_{\nu}$ is the spectral absorption coefficient, and $J_{b, \nu}$ is the spectral emissivity of black body. The transient term in Eq. (1) is omitted because the radiative process occurs with the speed of light rather than with the characteristic velocity of the flow. No scattering is assumed, because at typical temperatures in the shock layer the concentration of scattering centers is vanishingly small.

The formal solution of the radiation transfer equation along selected direction $s$ eliminates the angular dependence, and under the assumption of LTE the radiation intensity $J_{\nu}(s)$ is obtained in a closed form:

$$
J_{\nu}\left(\tau_{0}\right)=\int_{\tau_{0}}^{\tau} J_{b, \nu}\left(\tau_{1}^{\prime}\right) e^{-\left(\tau-\tau^{\prime}\right)} \mathrm{d} \tau^{\prime}
$$

where $\tau$ is the optical thickness of the participating media, and $\tau_{0}$ and $\tau_{1}$ are the optical thickness at the beginning and at the end of the interval $\left[\tau_{0}, \tau_{1}\right]$. The one-side radiation flux density at the surface of the space vehicle is then computed as

$$
W_{\nu}(s)=\int_{2 \pi} \Omega J_{\nu}(s, \Omega) \mathrm{d} \Omega
$$

The VF approach uses Eqs. (2) and (3) to obtain the flux density in two- and three-dimensional geometries. An emitting volume $V_{i}$ and receiving surface $A_{j}$ are shown in Fig. 1 . The VF of the volume $V_{i}$ with the respect to the surface $A_{j}$ is given by

$$
F_{i, j}=\frac{B_{i, j}}{V_{i}} \int_{A_{j}} \int_{V_{i}} T_{i, j, \nu} \frac{\cos \theta_{i, j}}{4 \pi r_{i, j}^{2}} \mathrm{~d} V_{i} \mathrm{~d} A_{j}
$$

where $B_{i, j}$ accounts for the shadowing between $A_{j}$ and $V_{i}, J_{i, \nu}$ is the spectral emissivity of the volume $V_{i}$, and $T_{i, j, \nu}$ is the attenuation 


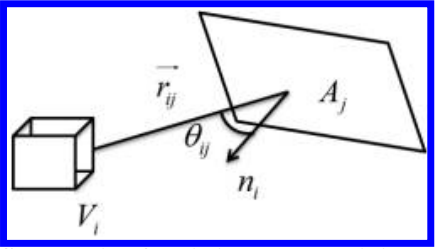

Fig. 1 Schematic of emitting and receiving elements.

factor along the distance $r_{i, j}$ dependent on the wavelength. Equation (4) is valid for any size and mutual location of $V_{i}$ and $A_{j}$.

The spectral radiation flux density at the surface $A_{j}$ is then calculated as

$$
W_{i, j, \nu}=\iint_{A_{j}} \int_{V_{i}} T_{i, j, \nu} B_{i, j} \frac{J_{i, \nu}}{A_{j}} \frac{\cos \theta_{i, j}}{4 \pi r_{i, j}^{2}} \mathrm{~d} V_{i} \mathrm{~d} A_{j}
$$

where $J_{i}$ is the spectral emissivity of the volume $V_{i}$. If the sizes of $V_{i}$ and $A_{j}$ are much smaller than $r_{i, j}$, then Eq. (4) can be simplified, as it was done in [25]:

$$
F_{i, j, \nu}=B_{i, j} T_{i, j, \nu} \frac{\cos \theta_{i, j}}{4 \pi r_{i, j}^{2}} A_{j}
$$

Equation (6) is valid only when relations

$$
\begin{aligned}
& r_{i, j} \gg \sqrt[3]{V_{i}} \\
& r_{i j} \gg \sqrt{A_{j}}
\end{aligned}
$$

are held. However, each pair of volume and surface can be divided into smaller subelements in such a way that Eqs. (7a) and (7b) are valid. A pair of subelements has an individual $\overline{\mathrm{VF}}$, but emitting properties and attenuation factor of all subelements belonging to the same element are identical. Thus, the spectral flux density emitted by the volume $V_{i}$ that reaches the surface $A_{j}$ is calculated as

$$
W_{i, j, \nu}=\frac{F_{i, j, \nu}}{A_{j}} \int_{V_{i}} J_{i, \nu} \mathrm{d} V_{i}
$$

When the volume $V_{i}$ is isothermal with the constant absorption coefficient $\kappa_{i}$, Eq. ( $\underline{8}$ ) can be simplified further:

$$
W_{i, j, \nu}=\frac{F_{i, j, \nu}}{A_{j}} J_{i, \nu} V_{i}
$$

The spectral radiation flux density at the surface $A_{j}$ is obtained by integration over all elements $V_{i}$, visible to $A_{j}$. Now, if the database of temperature, pressure, and species concentrations is available at nodes of either structured or unstructured grid, the spectral flux density can be calculated as $W_{j, \nu}=\sum_{j} W_{i, j, \nu}$. The integral flux density is found by integration of $W_{j, \nu}$ over the entire range of wavenumber.

The attenuation factor $T_{i, j, \nu}$ is given by the Beer-LambertBouguer law as an integral of the spectral absorption coefficient along the geometrical path $\left[s_{0}, s_{1}\right]$, corresponding to the optical interval $\left[\tau_{0}, \tau_{1}\right]$ :

$$
T_{i, j, \nu}=\exp \left(-\int_{s_{0}}^{s} \kappa_{\nu}(s) \mathrm{d} s\right)=\exp \left(-\tau_{\nu}\right)
$$

When the absorption between emitting and receiving elements is small, that is, $\tau_{\nu} \ll 1$, the attenuation factor virtually turns to unity.

If the flowfield is axisymmetric, it is possible to integrate Eq. () over the azimuthal angle $\varphi \in[0,2 \pi]$ of the cylindrical system of coordinates $(r, z)$, shown in Fig. 2 . Emitting elements that are located

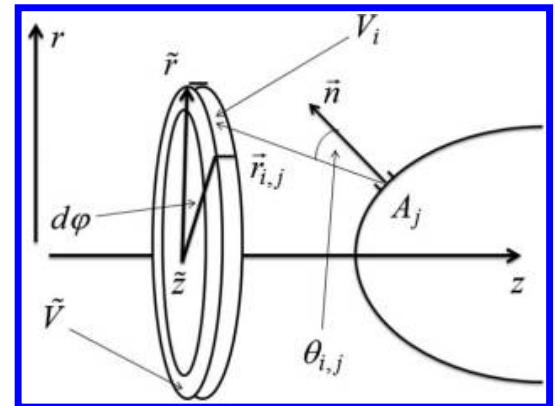

Fig. 2 Schematic of the VF approach for the reentry environment.

on the same distance $\tilde{r}$ from the axis of symmetry and have the same axial coordinate $\tilde{z}$ have the same $J_{b, \nu}$ and $\kappa_{\nu}$. Hence, the VF can be established for the toroidal element $\tilde{V}$, as it is shown in Fig. 2. Taking into account Eq. (4) and integrating over $\varphi$ within the cylindrical volume $\tilde{V}=2 \pi \tilde{r} \Delta \overline{\tilde{r}}$, one can obtain spectral radiation flux that comes from the element $\tilde{V}$, formed by the element $V_{i}$, on the surface $A_{j}$ :

$$
W_{i, j, \nu}=\int_{0}^{2 \pi} \int_{A_{j}} B_{i, j}(\varphi) \frac{J_{i, \nu}}{A_{j}} \frac{\cos \theta_{i, j}(\varphi)}{\pi r_{i, j}^{2}(\varphi)} T_{i, j}(\varphi) \tilde{r} \Delta \tilde{r} \mathrm{~d} A_{j} \mathrm{~d} \varphi
$$

where $B, T, \theta, I$, and $r$ are dependent on $\varphi$ unless the receiving surface $A_{j}$ is located on the axis of symmetry.

Shadowing factor $B_{i, j}$ varies from zero to unity. For the intermediate value of $B_{i, j}$, the surface $A_{j}$ is irradiated only by the fraction of $\tilde{V}$. Equation (11) provides the spectral flux density at the surface $A_{j}$ from the toroid formed by the volume $V_{i}$.

It is of interest to obtain an analytical expression of the integral in Eq. (11). In the case of an axisymmetric flowfield, the radiation intensity does not depend on azimuthal angle and can be taken out of the integral. It was found that the remainder can be expressed in elliptic integrals of first and second kind. The latter can be conveniently calculated by using either tabulated values or approximate methods [26]. The final derivation and resulting equations are given in the test case section.

In the case of three-dimensional geometry it is not possible to obtain an analytic form for the VF because the temperature and optical path vary with the azimuthal angle $\varphi$. The radiation flux density has to be calculated via direct numerical integration of Eq. (11):

$$
W_{i, j, \nu}=\sum_{k=1}^{N \varphi-1} \int_{A_{j}}\left(B_{i, j} \frac{J_{i, \nu}}{A_{j}} \frac{\cos \theta_{i, j}}{\pi r_{i, j}^{2}} T_{i, j} \tilde{r} \Delta \tilde{r}\right)_{\varphi_{k+1 / 2}} \mathrm{~d} A_{j}\left(\varphi_{k+1}-\varphi_{k}\right)
$$

where subscript $\varphi_{k+1 / 2}$ means the average of integrand on the interval $\left[\varphi_{k}, \varphi_{k+1}\right]$, total azimuthal angle is divided into $N_{\varphi}-1$ sectors, and $B_{i, j}$ accounts for the shadowing between element $A_{j}$ and the sector of the volume $\tilde{V}$, formed by $V_{i}$ and sector $\left[\varphi_{k}, \varphi_{k+1}\right]$ and defined by $\operatorname{sign}(\cos \theta)$. Direct numerical integration in Eq. (12) increases the cost of radiation model. In the present paper the comparison between Eqs. (11) and (12) is provided to shed light on the computational efficiency of the $\bar{V} F$ approach.

\section{B. Ray-Tracing Method}

The RTM has been extensively applied previously $[\underline{7}, 21,27]$ to obtain the radiation flux to the surface of a space vehicle. The key feature of the RTM is the direct angular integration of radiation intensity in the form given by Eq. (2). Because the mainfold integration of Eq. (3) over the angular variables is invoked, the RTM is extremely prohibitive. The RTM is extremely prohibitive because of the multiple integration over angular and spatial variables, required to estimate the left-hand side of Eq. (3).

Assuming that the LTE is valid, the numerical solution of Eq. (2) can be written in the following form: 


$$
J_{\nu}\left(\tau_{\nu}\right)=\sum_{k=1}^{N-1} \tilde{J}_{b, \nu} e^{-\left(\tau_{\nu, k}-\tau_{\nu}\right)}\left(\tau_{\nu, k+1}-\tau_{\nu, k}\right)
$$

where the interval $\left[s, s_{0}\right]$ along the ray $s$ is divided into $N-1$ intervals, and $\tilde{J}_{b, \nu}$ is the average spectral emissivity of the black body of the interval $\left[\tau_{k}, \tau_{k+1}\right]$. The summation in Eq. (13) is held along the entire path of the photon emitted from the point with coordinate $s^{\prime}$ until it reaches the surface. Typically, for the numerical estimation of Eq. (13) the black body emissivity is chosen as the arithmetic mean of $J_{b, \nu}$ at the end points of the interval $\left[\tau_{k}, \tau_{k+1}\right]$.

The resulting spectral flux density is given by Eq. (3). The angular integration in Eq. (3) can be performed using the conventional trapezoidal rule:

$$
\begin{gathered}
W_{\nu}(s)=\int_{0}^{2 \pi} \mathrm{d} \psi \int_{0}^{\pi / 2} J_{\nu}(s, \Omega) \cos \theta \sin \theta \mathrm{d} \theta= \\
=\sum_{m=1}^{N_{\psi}-1} \mathrm{~d} \psi \sum_{n=1}^{N_{\theta}-1} J_{\nu}\left(\Omega_{m, n}\right)\left[\sin \theta_{n+1} \cos \theta_{n+1}-\sin \theta_{n} \cos \theta_{n}\right] \mathrm{d} \theta
\end{gathered}
$$

where $d \psi=\psi_{n+1}-\psi_{n}$ and $d \theta=\theta_{n+1}-\theta_{n}$ are the increment of angles of local spherical system of coordinates on the surface of the space vehicle (shown in Fig. 3).

Equation (14) implies that the radiative properties on the tracing ray must be known. Because the grid on the tracing ray does not generally correspond to the node location of the gasdynamic grid, the data interpolation is required. The NNS procedure is involved to determine the node of the gasdynamic grid that is nearest to the current location on the tracing ray. Because the summation in Eq. (13) is performed in a consecutive manner, it is more efficient to search for the nearest neighbor of point $k$ in a vicinity of the previous point $k-1$ on the same tracing ray, for which the nearest neighbor has already been determined. This method performs a search of the nearest neighbor in a consecutive manner and is referred to as the consecutive nearest neighbor search (CNNS) algorithm [28]. The CNNS method is one of the most efficient methods to determine the optical thickness along the tracing ray. An alternative algorithm is to search for the nearest neighbor over all nodes of the gasdynamic grid. This method is often referred to as a brute search algorithm.

The schematic of the search domain is shown in Fig. 4. If the nearest neighbor is known for point 1 , the nearest neighbor for point 2 is located in the area shown with the dashed line. The improvement in the efficiency using CNNS algorithm compared with the brute search is significant. For the purpose of comparison the present paper adopts both the CNNS and brute NNS algorithms.

The RTM uses $N_{\psi}=41$ rays in the azimuthal direction and $N_{\theta}=41$ rays in the radial direction. The distribution of integration points along the tracing ray is given by Gauss-Lobatto quadratures, previously applied to improve the accuracy of integration in Eq. (2) [21]. By clustering points at the bounds of the tracing ray, the number of points can be reduced by approximately a factor of four. The properties between the two nearest grid points are linearly interpolated. To understand the importance of this step, a set of calculations with no interpolation is performed. For the latter, the gas properties on the tracing ray are simply assigned as the properties of the nearest node of the gasdynamic grid.

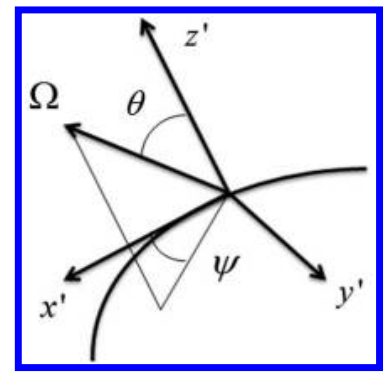

Fig. 3 Spherical system of coordinates on the surface.

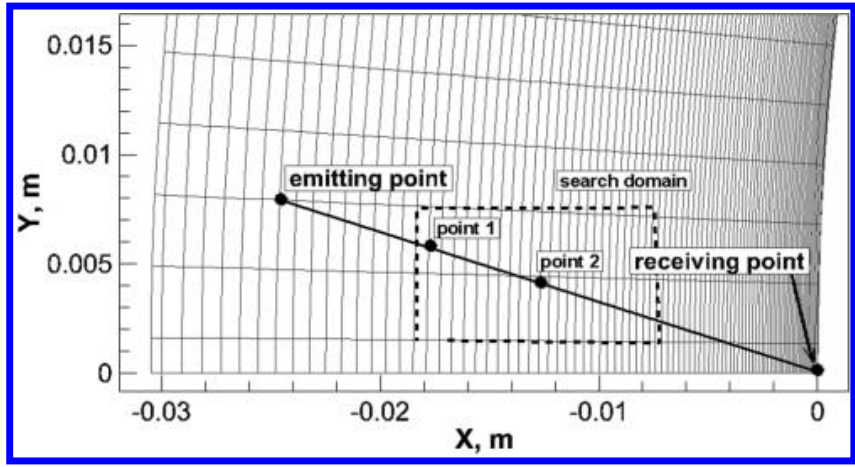

Fig. 4 Schematic of the CNNS algorithm.

When the media between emitting and receiving elements is nearly transparent, the calculation of optical thickness can be omitted. It is then assumed that emitted photons reach the surface without attenuation. In the case of the RTM, the NNS is still required to determine the properties of the emitting point along the tracing ray (see Fig. 4). When the VF approach is applied, this step is omitted, because the emitting volume is built on the node of the gasdynamic grid with originally computed properties. This fact is an additional advantage of the VF approach.

\section{Physical Model}

The solution of gasdynamic equations provides the database for determining the optical and radiation properties and precedes the implementation of the radiation transport model. Simulation of highenthalpy hypersonic flow involves physical and chemical models at different molecular scales. To comply with previous models of nonequilibrium flows, the present work adopts a three-temperature model to describe the thermal nonequilibrium behind a shock wave. The vibrational and electron-electronic modes are treated separately from each other for the accurate description of electronic temperature and concentrations behind the shock wave, because the reaction of impact ionization appears to be the second important mechanism of the source of free electrons [28]. Governing system of equations employs the Navier-Stokes equations coupled with the trans-rotational energy conservation law, chemical species mass conservation equations, and a model for the energy exchange between integral degrees of freedom. The resulting multiphysical model is described in detail in [28].

The spectral radiation model contains 96 groups of nonuniform width and covers interval of wavenumber from $10^{3}$ to $10^{5} \mathrm{~cm}^{-1}$. The absorption coefficient depends on the temperature, pressure, and gas composition. An averaging procedure is applied to obtain spectral absorption coefficient within each spectral group [6]. The radiation transfer model is decoupled from the gasdynamic equations due to the small ratio of radiative flux to the small Goulard parameter [29].

\section{Test Case}

The reentry of the RAM-C II probe is chosen to investigate the efficiency and accuracy of radiation transfer models. This choice of the flight experiment is caused by a significant amount of experimental data and theoretical investigations on the plasma composition behind the shock wave conducted over the past four decades. At the same time, the reentry velocity of the RAM-C II probe is less than that of superorbital probes. This fact allows avoiding the coupling of gasdynamics and radiation transfer.

Parameters of the ambient flow at three altitudes are shown in Table 1 . The velocity of the probe at the beginning of descent is $7.5 \mathrm{~km} / \mathrm{s}$ and the Goulard parameter does not exceed $10^{-3}$. The flow regime spans from a significant nonequilibrium at the altitude of $81 \mathrm{~km}$ to a nearly equilibrium at the altitude of $61 \mathrm{~km}$.

To ensure the convergence of results, the flowfield is computed on two grids with the dimensions of $(186 \times 94)$ and $(326 \times 186)$ nodes, referred to as coarse and fine grid and shown in Fig. 5 . The grid number density used in present calculations is equal to or exceeds the 
Table 1 Parameters of ambient flow

\begin{tabular}{lccrrc}
\hline \hline Altitude, $\mathrm{km}$ & $M$ & $T_{\infty}, \mathrm{K}$ & \multicolumn{1}{c}{$R e$} & $P_{\infty}, \mathrm{Pa}$ & $K n$ \\
\hline 61 & 23.9 & 254.80 & 19,500 & 19.5 & $1.8 \times 10^{-3}$ \\
71 & 25.9 & 219.58 & 6,280 & 4.9 & $6.1 \times 10^{-3}$ \\
81 & 28.3 & 198.64 & 1,580 & 1.0 & $2.8 \times 10^{-2}$ \\
\hline \hline
\end{tabular}

typical resolution used for this problem [23]. The VF approach is tested on both of these grids.

To apply the VF approach to the axisymmetric flow, the shape and location of emitting elements must be established. The distinct advantage of the VF approach is in the fact that such elements can be formed on a basis of an existing computational grid that was used originally to compute temperature, pressure, and species composition.

For each grid point $\left(x_{0}, y_{0}\right)$, shown in Fig. 6, the emitting volume $\tilde{V}$ with the quadrangular cross section $\mathrm{AB} \overline{C D}$ is constructed. The emitting volume $\tilde{V}$ is a toroid with the cross-sectional area $S_{\mathrm{ABCD}}$ and radius $\tilde{r}$. Because $\tilde{V}$ contains only a single grid point, the properties of radiating media within $\tilde{V}$ are assumed constant and equal to the properties at grid point $\left(x_{0}, y_{0}\right)$. The schematic of the emitting volume and receiving surface is shown in Fig. 2. Substituting the trivial geometric expression for the radius vector $r_{i, j}$ and angle $\theta_{i, j}$ into Eq. (5), the expression for the radiation flux density can be obtained:

$W_{i, j, \nu}=J_{i} S_{\mathrm{ABCD}} \tilde{r} \int_{\varphi_{1}}^{\varphi_{2}} T_{i j} \frac{\left(x_{i}-x_{n}\right) n_{x}-y_{n} n_{y}+R_{i} n_{y} \cos \varphi}{\left(\left(x_{i}-x_{n}\right)^{2}+R_{i}^{2}+y_{n}^{2}-2 R_{i} y_{n} \cos \varphi\right)^{3 / 2}} \mathrm{~d} \varphi$

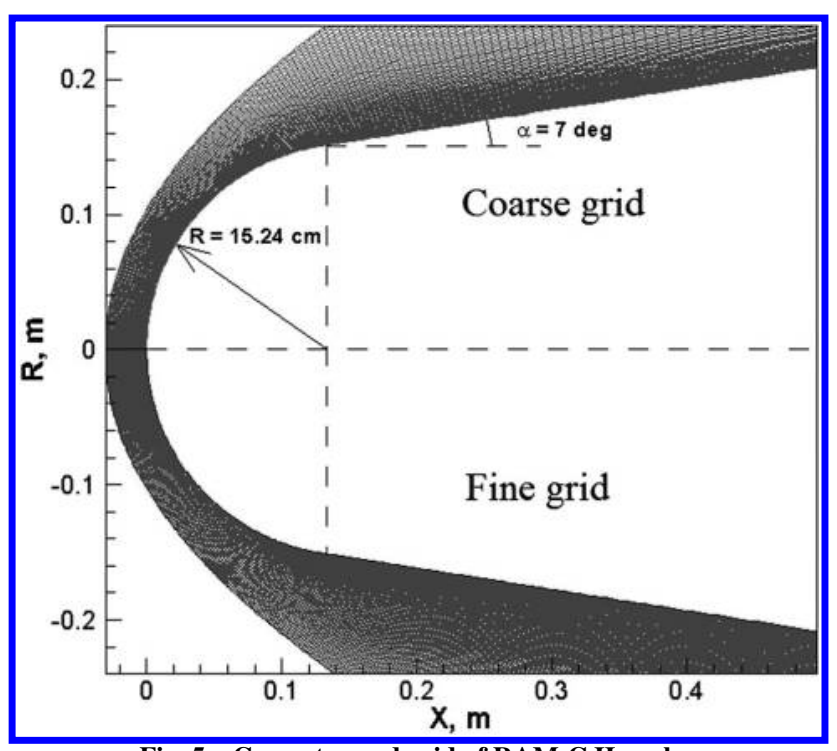

Fig. 5 Geometry and grid of RAM-C II probe.

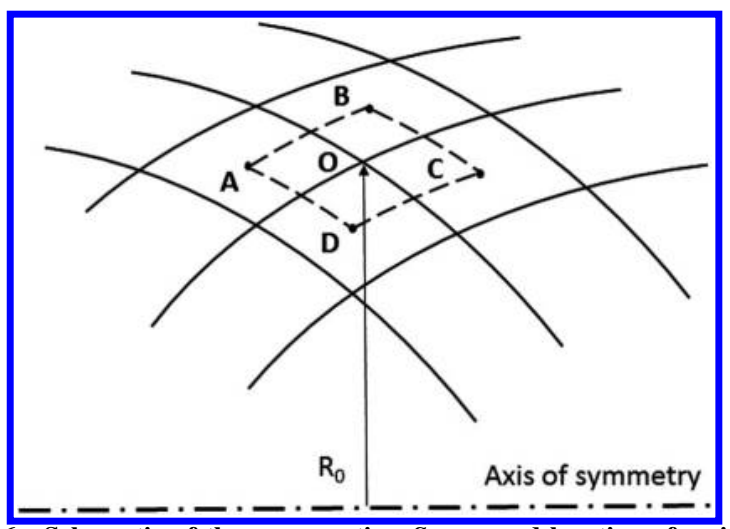

Fig. 6 Schematic of the cross section $S_{\mathrm{ABCD}}$ and location of emitting volume $V_{i}$. where $\left(n_{x}, n_{y}\right),\left(x_{n}, y_{n}\right)$ are coordinates of the unit normal vector $\boldsymbol{n}$ to $A_{j}$ and the center of $A_{j}$. The shadowing factor $B_{i, j}$ in Eq. (15) is replaced by integration over the azimuthal angle $\varphi$ within the interval $\left(\varphi_{1} ; \varphi_{2}\right)$ when the volume $\tilde{V}$ is visible for $A_{j}$. The azimuthal angles $\varphi_{1}$ and $\varphi_{2}$ can be easily computed for two-dimensional axisymmetric geometry. For three-dimensional geometry the shadowing factor $B_{i, j, k}$ of the segment $k$ simply takes the Boolean value. In case of no shadowing, $\varphi_{1}=0$ and $\varphi_{2}=2 \pi$.

The integral in Eq. (15) cannot be expressed in the elementary functions. However, a semi-analytical expression is available by mean elliptic integrals of the first and second kind. The numerical calculation of elliptic integrals is a well-known problem and efficient solutions are available [26]. The integral, used in present computations, is given by Eq. ( 16 ). The right-hand side of Eq. (15) can be transformed to the left-hand side of Eq. (16), while the analytical expression for the resulting flux density is given by right-hand side Eq. (16). The total flux on the surface element $A_{j}$ can be obtained by summation in Eq. (15) over all radiating volumes $V_{i}$

$$
\begin{aligned}
& \int \frac{(\cos (x)+a)}{(\cos (x)+b)^{3 / 2}} \mathrm{~d} x=C+A(b-a) \sin (x) \\
& -A\left[(b+1)(b-a) \sqrt{\frac{b+\cos (x)}{b+1}} E\left(0.5 x \mid \frac{2}{b+1}\right)\right] \\
& +A\left[\left(b^{2}-1\right) \sqrt{\frac{b+\cos (x)}{b+1}} F\left(0.5 x \mid \frac{2}{b+1}\right)\right]
\end{aligned}
$$

where $A=2 /\left[\left(b^{2}-1\right) \sqrt{b+\cos (x)}\right]$.

Equation (15) is derived under the assumption that the surface element $A_{j}$ and volume element $V_{i}$ are far enough from each other and so the radius vector and angle $\theta_{i, j}$ in Eq. ( $\underline{8}$ ) can be assumed constant during the integration. In the case of closely spaced elements $V_{i}$ and $A_{j}$, an additional subdivision into smaller elements may be necessary [16]. This situation takes place when attempting to account for the contribution of elements located close to the surface. Although the emission near the wall is typically much lower in comparison with the contribution of the shock layer, the absorption of radiation in the near-surface region can be significant. It will be shown further that for the flow at low altitudes the absorption of radiation is noticeable and an accurate treatment of closely spaced elements is required. Once again, Eqs. (15) and (16) provide a semi-analytical expression for the spectral flux density on the surface of a space vehicle resulting in twodimensional axisymmetric geometry, assuming emitting but not absorbing media.

Analytical expressions, given by Eqs. (15) and (16), are applicable also on unstructured grids of arbitrary topology, because the present derivation does not assume any specific shape of emitting element. Moreover, the general expressions of the VF approach, given by Eqs. (11) and (12), hold for two- and three-dimensional unstructured grids too. However, the algorithm of NNS, if invoked, should be adapted for the appropriate grid topology.

\section{Results}

\section{A. Simulation of Flowfield}

The reentry of the RAM-C II probe serves as a classical tool for the validation of nonequilibrium flowfield model. During the reentry, the critical number density of electrons was measured by a set of microwave reflectometers at four locations on the surface of the probe [22]. This experimental data provides a background for the validation of gasdynamic model, adopted in the present work.

Translational and $N_{2}$ vibrational temperatures along the axis of symmetry, computed for three altitudes, are shown in Fig. 7. At high altitudes the vibrational temperature is substantially lower than gas kinetic temperature for the entire relaxation zone and reaches the equilibrium only in the vicinity of the surface. The shock front is smeared, which is due to the relatively large mean free path. As shown below, the rarefied flowfield at the altitude of $81 \mathrm{~km}$ results in a very minor absorption of radiation. 


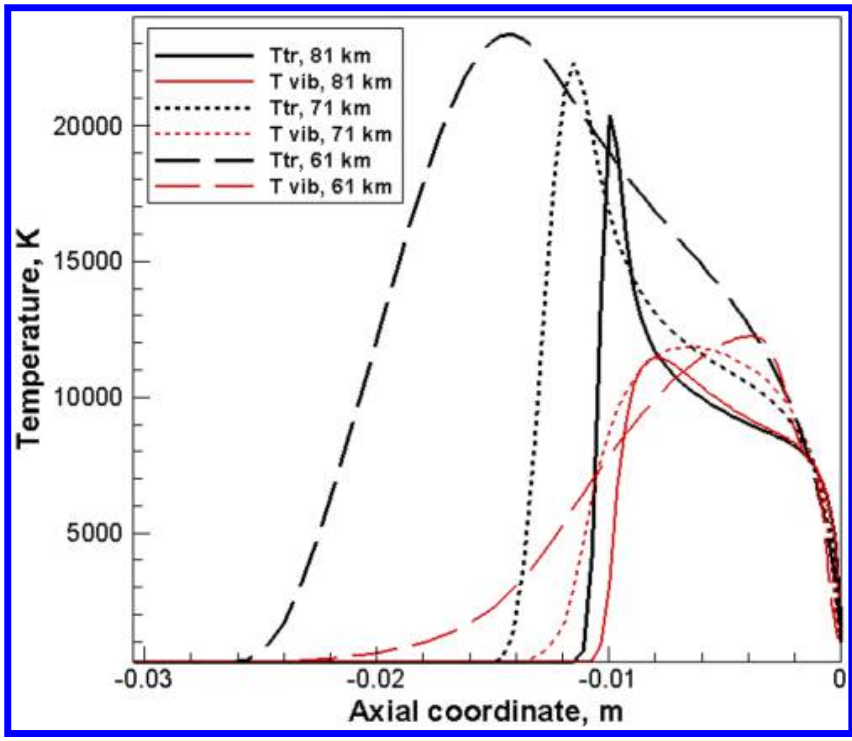

Fig. 7 Translational and $N_{2}$ vibrational temperatures.

The flowfield at the altitude of $61 \mathrm{~km}$ possesses a certain degree of nonequilibrium as well. However, the equilibration of internal temperature under these conditions occurs at the much smaller scale than that at the altitudes of 71 and $81 \mathrm{~km}$. The shock standoff distance and the peak temperature decrease with the altitude. The latter is explained by a more pronounced dissociation of species behind the shock wave at increased density of the ambient flow.

The critical number density of free electrons is compared with the experimental data in Fig. $\underline{8}$ for the altitudes of 61, 71, and $81 \mathrm{~km}$. Solid curves correspond to the present results; symbols describe the measured concentrations. The electron number density strongly depends on the flight conditions and quickly diminishes toward the base of the vehicle. A satisfactory agreement between computed data and experiment is established. A larger discrepancy of theoretical and experimental results for the altitude of $61 \mathrm{~km}$ may be explained by a significant sensitivity of the electron number density to the chemical model, as stated in [30].

The database of temperature, pressure, and gas composition is generated for each flight condition and is used to calculate the multigroup absorption coefficient $\kappa_{\nu}$ at each point of the gasdynamic grid, shown in Fig. 5. A sample of spectral absorption coefficient behind the shock wave is shown in Fig. 9 for the altitudes of 61, 71, and $81 \mathrm{~km}$. There is a significant drop in the absolute value of $\kappa_{\nu}$ for studied range of altitudes as well as for the wavenumber of interest. Namely, the absorption coefficient is several orders of magnitude

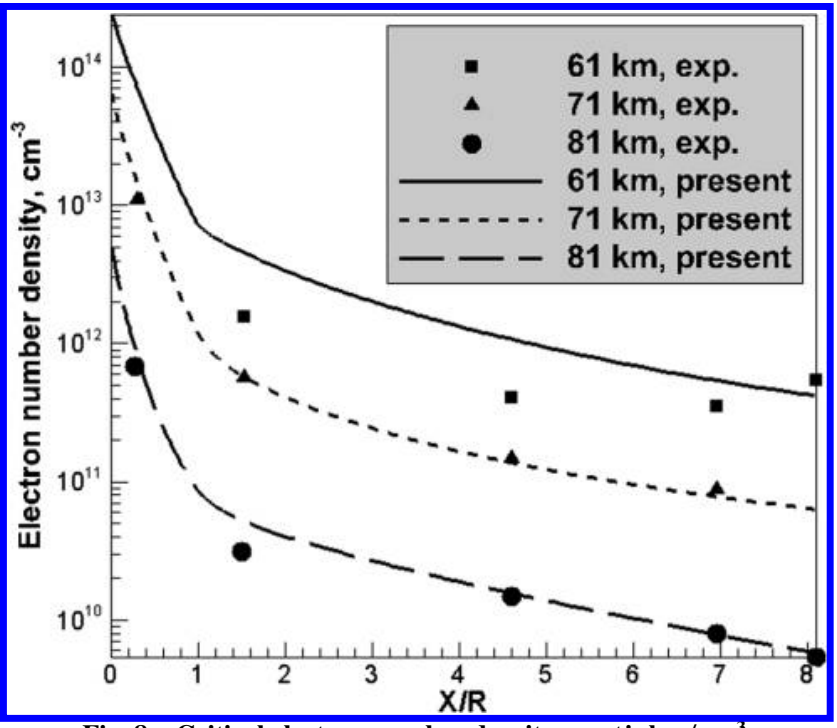

Fig. 8 Critical electron number density, particles $/ \mathrm{cm}^{3}$.

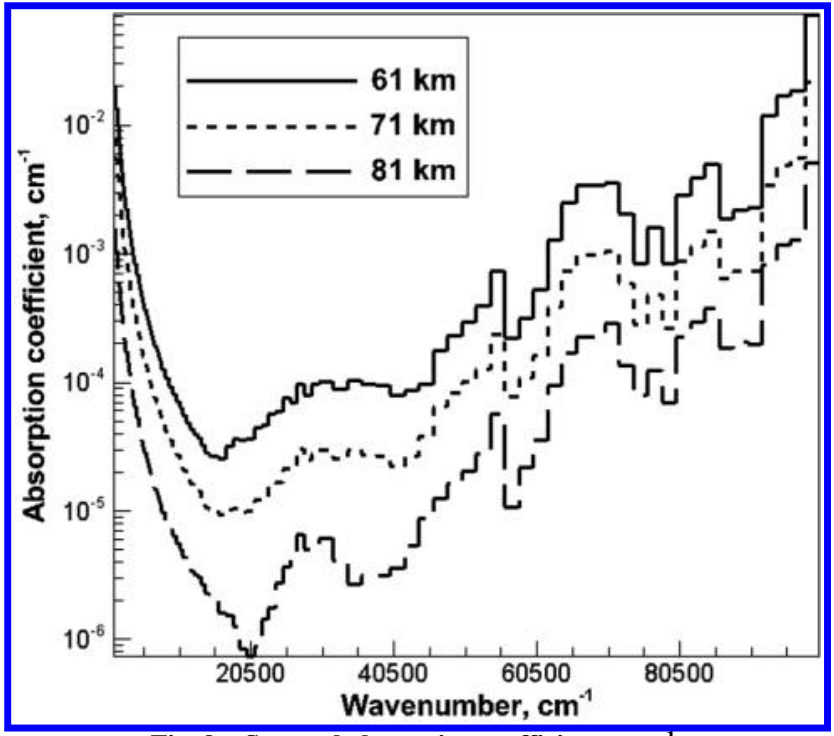

Fig. 9 Spectral absorption coefficient, $\mathbf{c m}^{-1}$.

lower for the altitude of $81 \mathrm{~km}$. Thus, the case of radiation transfer in the optically thin media should receive a special attention.

\section{B. Convergence of Radiation Flux Density}

When absorption of radiation is neglected, the VF approach uses Eq. (15). The attenuation factor $T_{i, j}$ is equal to unity for each emitting element $V_{i}$, and no approximation of gas properties is needed to calculate optical thickness of the media. However, in order to properly account for the emission from the volumes closely spaced to the wall, the refinement is needed. The decision on the refinement can be made based on the ratio of the element size and its distance to the surface element. When this ratio is small, say, 0.1 , no refinement is needed. In the opposite situation, each gasdynamic cell is divided into $m$ subelements in $i$ and $n$ subelements in $j$ directions, where $i$ and $j$ are the indices of two-dimensional gasdynamic grid. Following this procedure, converged results are obtained taking into account even grid elements adjacent to the surface. For the VF approach the cost of the refinement is significantly smaller than the cost of calculation of the emitting properties. In the present case, the refinement takes no more than $10 \%$ of the total computational time.

Refined and nonrefined VFs in the vicinity of the front stagnation point are shown in Fig. 10. The refinement does not introduce any visible changes for the elements at the shock layer $(x \approx 0.9 \mathrm{~cm})$.

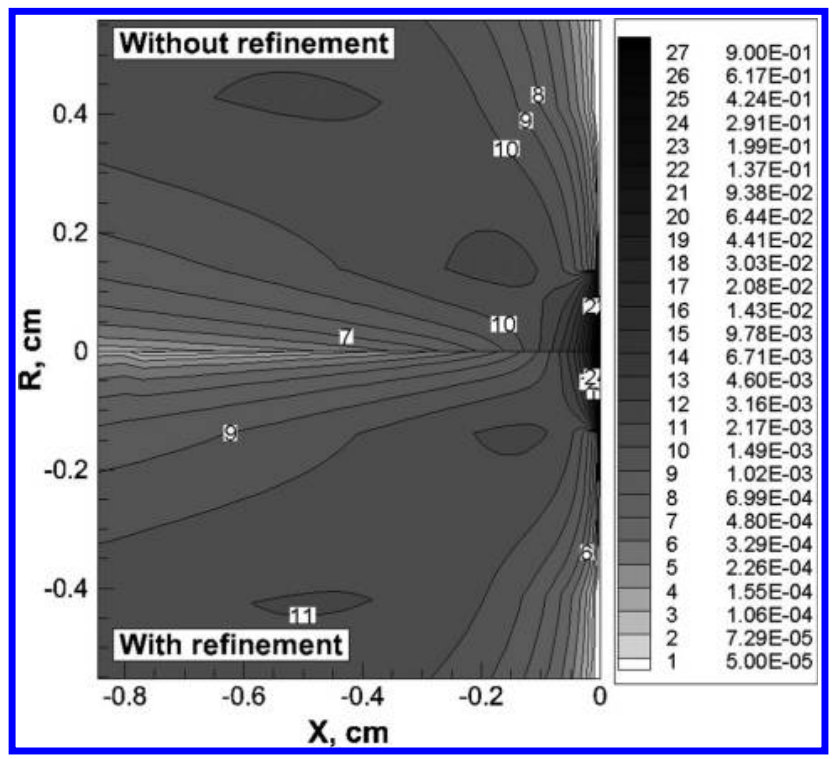

Fig. 10 View factor for the stagnation point. 


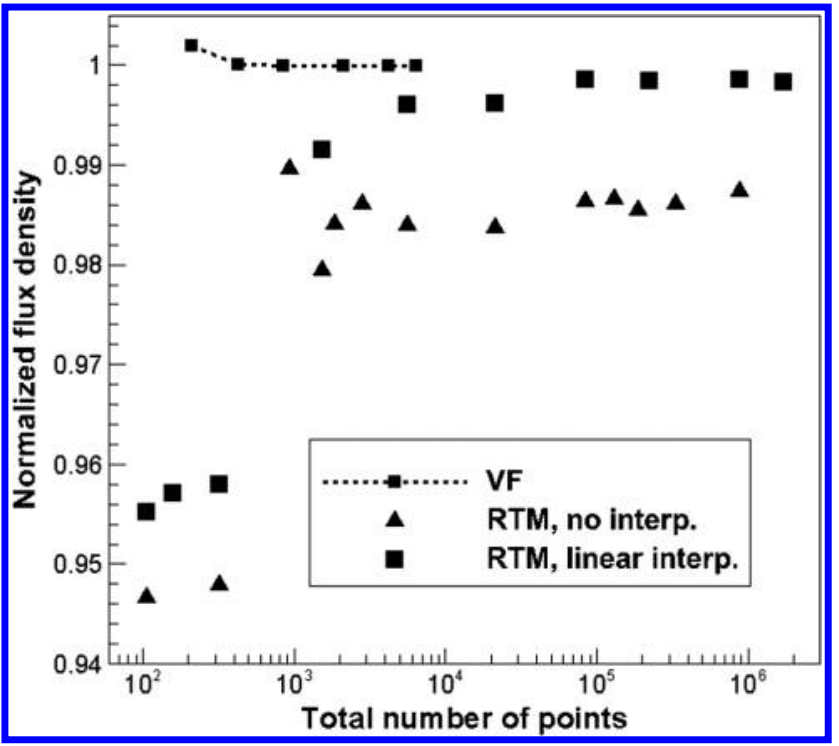

Fig. 11 Convergence of VF and RTM.

However, the VF for elements next to the surface can be overestimated by a factor of $2-3$. Meanwhile, the VF in axisymmetric geometry is a nonmonotonic function of the distance. The VF reaches a local maximum at some distance from the surface, due to the large $\tilde{r}$ for these elements.

Convergence of the flux density for the front stagnation point is presented in Fig. 11 for the altitude of $81 \mathrm{~km}$. The RTM is applied using the linear approximation (square symbols) of properties between the two nearest grid points as well with no approximation (triangular symbols). The total number of points corresponds to the overall amount of integration points along each ray for the RTM. In the case of the VF approach, given by the dashed line, the total number of points defines the overall amount of subelements used in the calculation.

The number of subelements required for a converged solution is in the order of $2 \times 10^{2}$ for the VF approach. The RTM generates a converged solution only at a very high number of points, unacceptable in the everyday practice. The small, but nonvanishing difference between the VF approach and RTM is due to the inaccuracy in the properties approximation in the vicinity of the shock wave. In this sense, the utilization of the original grid by the VF approach becomes a clear advantage.

\section{Radiation Flux Density}

The case when absorption is taken into account makes utilization of the VF approach more complicated and expensive. The optical thickness has to be calculated for each pair of emitting and receiving elements, and thus the search on nearest neighbor is involved. The points along the distance between emitting volume and receiving surface are equally distributed and gas properties are evaluated at their location. To avoid an excessive number of points for a short distance (when emitting element is located near the surface), the available number of nodes of gasdynamic grid is estimated. For a point with grid index $\left(i_{s}, j_{s}\right)$ at the surface and emitting volume $\left(i_{v}, j_{v}\right)$, the number of points is estimated as $\max \left(\left|i_{s}-i_{v}\right|,\left|j_{s}-j_{v}\right|\right)$. This step saves a significant amount of computational resources.

The normalized flux density at three altitudes, considered in Table 1, is presented in Figures 12-14. The horizontal coordinate is equal to the ratio of surface length to the radius of hemispherical cap, starting from the front stagnation point. Figures 12-14a describe the heating of the front shield, while Fig. 14b presents the heating of the leeward surface. The results of the TS approximation include the absorption and are given by the short dashed line; the RTM and VF approach are shown by solid and long dashed lines, respectively. Symbols correspond to the calculation without taking into account the absorption of radiation.

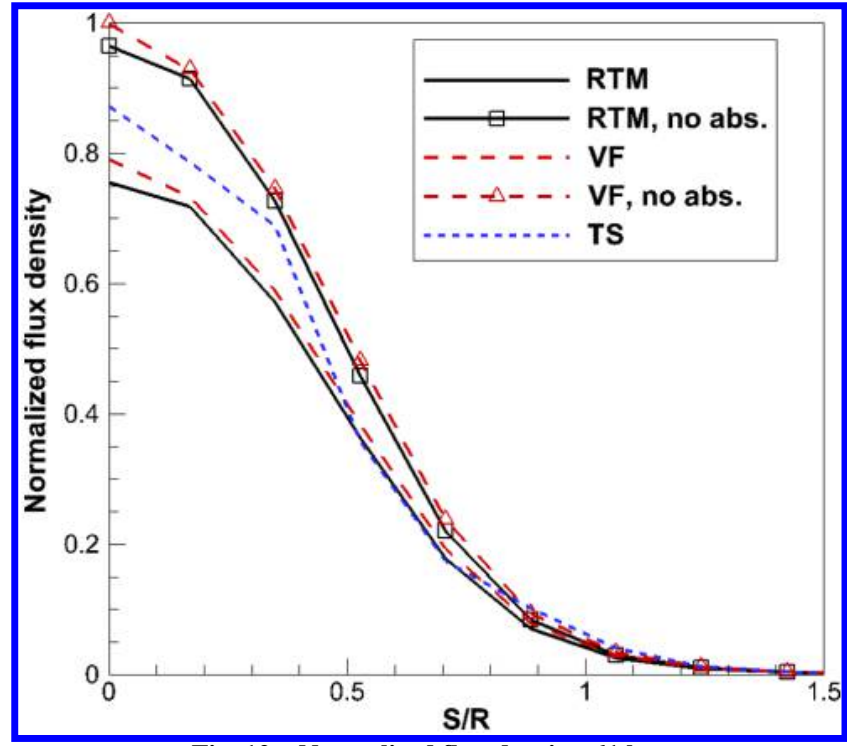

Fig. 12 Normalized flux density, $61 \mathrm{~km}$.

The TS approximation generally has unsatisfactory accuracy of the radiative flux density only on the hemispherical cap of the vehicle. The difference between the TS approximation and the RTM and the VF approach does not exceed $30 \%$ for the front surface, which is in the range of expected error due to the surface and shock layer curvature [25]. The TS approximate works better for lower altitudes when the mean free path is short and the shock is less smeared. The large underestimation of the flux density by the TS approximation at the afterbody is observed in Fig. 14b with a maximum of two times. This fact is explained by the shape of the shock wave that can no longer be approximated as an infinite slab.

The stagnation flux calculated by the RTM for both absorbing and transparent media is given in Table 2 . The radiation absorption at a given entry conditions plays an important role only for low-altitude points of trajectory. The unimportance of absorption at high altitudes can be explained by a relatively low density of the atmosphere. However, at the altitude of $61 \mathrm{~km}$, attenuation due to the absorption is more than $20 \%$ of the total contribution by the shock layer and has to be taken into account.

Overall, the RTM and VF approach reach a satisfactory agreement. The maximum of the normalized difference is not more than $5 \%$ for the forebody and $10 \%$ for the afterbody. The nonvanishing difference is caused by the grid-to-grid approximation of properties employed by the RTM, as it follows from Fig. 11 .

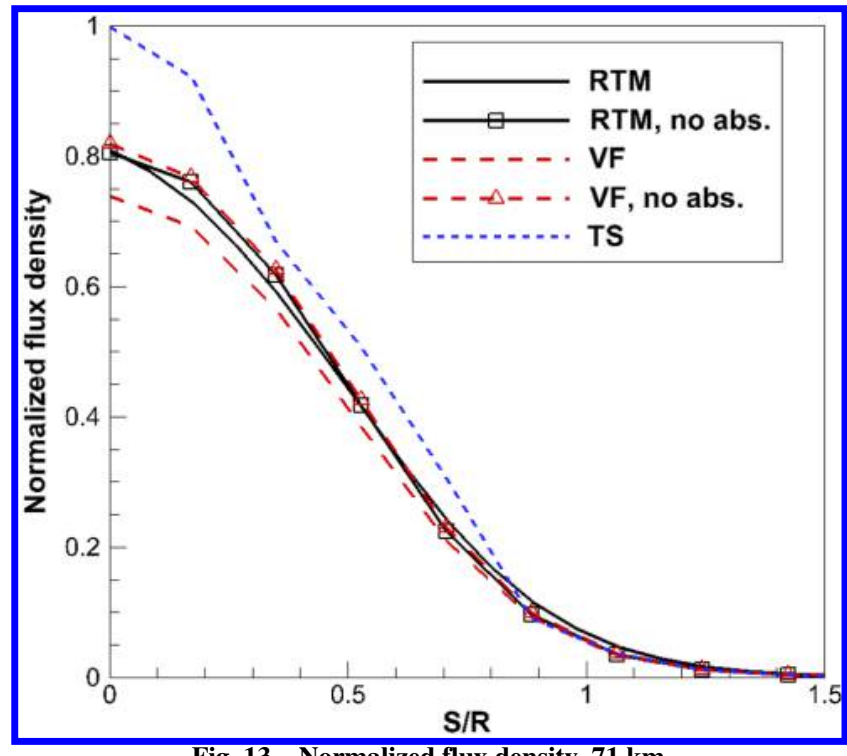



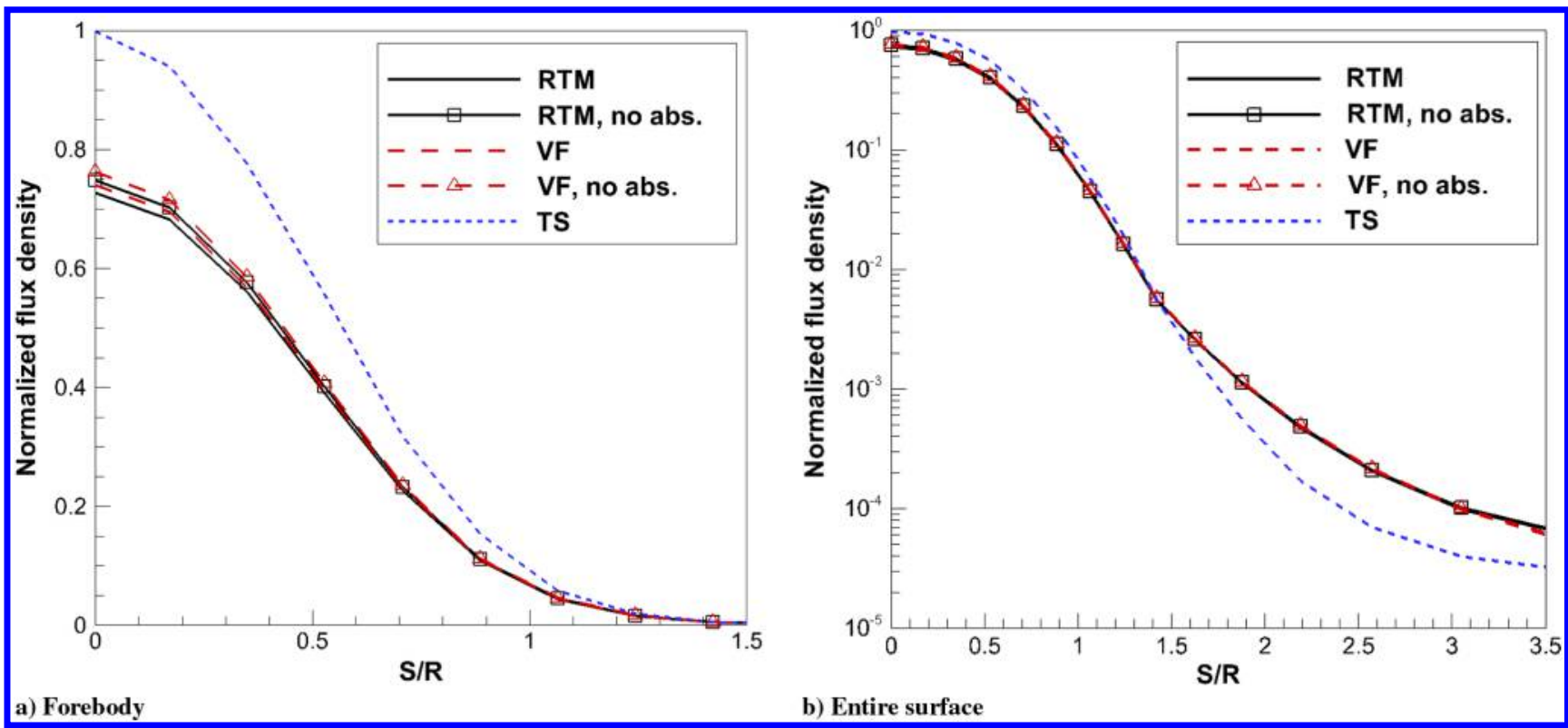

Fig. 14 Normalized flux density, 81 km.

The overall heating rates and the percentage of the radiation flux are presented in Table 3 . The convective component of flux density prevails over the radiation heating rate for all three altitudes of trajectory, which is an expected results for the reentry velocity below $10 \mathrm{~km} / \mathrm{s}$. Theoretical estimations of the RAM-C II convective flux are rarely reported in the literature. The heat flux for the altitude of $71 \mathrm{~km}$ ranges between 96 and $218 \mathrm{~W} / \mathrm{cm}^{2}$ [31]. This large uncertainty is caused, first of all, by the particular chemical model employed in calculations. Present convective heating rate at the altitude of $71 \mathrm{~km}$ falls in the acceptable range. To the authors' knowledge, the data, similar to that in Table $\underline{3}$, were not previously given in the open literature.

Radiative component of the flux decreases with the altitude from 20.67 to $8.39 \%$. It is worth to note that the absolute value of radiation flux can be possibly overestimated due to the assumption of local thermodynamic equilibrium. This situation is particularly important in the rarefied flows at altitude of $81 \mathrm{~km}$, where the deviation from thermal equilibrium is significant. However, the purpose of the present research is only to demonstrate the advantage of the VF approach as a radiation transfer model. Future investigation will be related to the coupling of the VF approach and a more accurate thermodynamic model.

\section{Efficiency of Radiation Model}

The computational efficiency of the RTM and VF approach is reported when radiation flux is calculated at the stagnation and afterbody points. Calculations are performed taking into account absorption in the real spectrum of high-temperature air as well as assuming

Table 2 Radiation flux density $\left(\mathrm{W} / \mathrm{cm}^{2}\right)$, with and without absorption taken into account

\begin{tabular}{lccc}
\hline \hline Altitude, $\mathrm{km}$ & With absorption & Without absorption & Relative difference \\
\hline 81 & 27.42 & 28.21 & 0.028 \\
71 & 32.60 & 36.06 & 0.096 \\
61 & 28.90 & 36.92 & 0.217 \\
\hline \hline
\end{tabular}

Table 3 Convective and radiation flux densities, $W / \mathrm{cm}^{2}$

\begin{tabular}{lcccc}
\hline \hline Altitude, flux, km & Convective & Radiative & Total & Rad./total, \% \\
\hline 61 & 305.59 & 28.90 & 344.49 & 8.39 \\
71 & 191.53 & 32.60 & 224.13 & 14.54 \\
81 & 105.20 & 27.42 & 132.62 & 20.67 \\
\hline \hline
\end{tabular}

that the gas is optically transparent. This numerical experiment aims to estimate an effect of attenuation on the computational cost of the radiation transfer model.

The cost of the VF approach at different numbers of subelements is presented in Table 4 for the altitude of $81 \mathrm{~km}$, neglecting the absorption of radiation. The number of subelements varies from $n=3=1$ (no refinement) to $n=m=5$ in $i$ and $j$ directions. For the latter, two grids $(186 \times 94$, coarse mesh $)$ and $(326 \times 186$, fine mesh $)$ are used. Comparison is performed for the point with surface index $i=1$ at the stagnation point $(x=0 \mathrm{~cm})$ and $i=171$ at the base $(x=112 \mathrm{~cm})$ of the vehicle. The reported times include the calculation of the VF as well as of the radiation properties.

The refinement of emitting elements has a minor effect on the efficiency of the VF approach. Indeed, the computational time increases by 2.7 and $5.1 \%$ for the stagnation point when the number of subelements is increased 5 and 25 times, respectively, indicating that the main effort is spent to obtain the emitting properties. The computational time at the afterbody is slightly large: 3.1 and $7.7 \%$, respectively, which is explained by a larger size of grid elements compared with the stagnation region. When absorption is taken into account, one can expect even less influence of the refinement on the overall efficiency of the VF approach.

The computational time and operation count for all three radiation models are presented in Table 5 . In all cases the absorption of radiation is taken into account if the opposite is not stated. To highlight the computational cost of the particular approach, the total count and optical properties count are reported separately. In the case of the RTM, calculations are performed for both consecutive and brute NNS algorithms.

It follows from Table 5 that the TS approximation is the most efficient and least accurate radiation transfer model. High efficiency of the TS approximation is due to the semi-analytical integration of the angular dependence of the radiation intensity using integralexponential functions [32]. However, it seems that for space vehicles with a small curvature of the surface, the TS approximation may generate misleading results. The computational time of the VF

Table 4 Required time (in seconds) of the VF approach for different numbers of subelements

\begin{tabular}{lcccc}
\hline \hline Subelements & $m=n=1$ & $m=5, n=1$ & $m=n=5$ & $m=n=5$ \\
\hline Grid point & Coarse mesh & Coarse mesh & Coarse mesh & Fine mesh \\
$i=1$ (stagnation) & 4.29 & 4.41 & 4.52 & 8.30 \\
$i=171$ (afterbody) & 28.51 & 29.42 & 30.83 & 72.56 \\
\hline \hline
\end{tabular}


Table 5 Time and number of operations for VF approach, RTM, and TS method, $(186 \times 94)$ grid

\begin{tabular}{lccc}
\hline \hline Method & Time, $\mathrm{s}$ & Total count & Properties count \\
\hline TS & 0.5 & $1.02 \times 10^{9}$ & $7.59 \times 10^{8}$ \\
VF, no absorption & 3.4 & $1.41 \times 10^{10}$ & $5.20 \times 10^{9}$ \\
VF, with absorption & $4.0 \times 10^{2}$ & $7.41 \times 10^{11}$ & $1.20 \times 10^{11}$ \\
RTM, CNNS & $5.2 \times 10^{2}$ & $1.02 \times 10^{12}$ & $8.09 \times 10^{11}$ \\
RTM, brute NNS & $1.8 \times 10^{3}$ & $4.48 \times 10^{12}$ & $6.86 \times 10^{11}$ \\
\hline \hline
\end{tabular}

Table 6 Computational time and operation count of VF approach and RTM, $(326 \times 186)$ grid

\begin{tabular}{lccc}
\hline \hline Approach/parameters & Time, $\mathrm{s}$ & Total count & Properties count \\
\hline VF, no absorption & 9.8 & $2.1 \times 10^{10}$ & $1.7 \times 10^{10}$ \\
VF, with absorption & $7.9 \times 10^{2}$ & $1.3 \times 10^{16}$ & $4.6 \times 10^{15}$ \\
RTM, CNNS & $4.2 \times 10^{3}$ & $8.87 \times 10^{16}$ & $4.14 \times 10^{16}$ \\
\hline \hline
\end{tabular}

approach is only 6.8 times larger than the TS approximation if no absorption is taken into account.

When compared with the RTM, the VF approach demonstrates significant savings in computational time. In the case of transparent media, the difference in the efficiency is 151.5 and 533.8 times when consecutive and brute NNS algorithms are used, respectively. An increased cost of the VF approach when absorption is taken into account is caused primarily by the cost of the calculation of the optical properties. This conclusion follows from the increase of the properties count by nearly the same factor as the total count of calculations. In this light the development of more efficient optical models is highly desired because it is a restriction factor of the overall efficiency.

The study of the efficiency is also performed for the fine mesh and presented in Table 6. The computational time of the VF approach increases linearly with the number of grid points along in one direction. On the contrary, the cost of the RTM increases faster than that for the VF approach. This is explained by the increased costs of the radiation transfer model, given by Eq. (2), regardless of the optical model. It follows from Tables 5 and 6 that the former shares nearly $80 \%$ of total computational time in the case of a coarse grid, and only $40 \%$ when a fine grid is used. In other words, the radiation transfer model based on the VF approach demonstrates less sensitivity in the cost to the grid refinement, compared with the RTM.

\section{Conclusions}

The radiation transfer model based on the view-factor (VF) approach is developed to solve the radiation transfer equation for the reentry flowfield. Newly derived equations for the radiation flux density are coupled to the gasdynamic code and are tested using the multigroup optical model of the air plasma. The radiation flux density on the surface of the RAM-C II space vehicle is obtained in the semianalytical form when the VF approach is applied in two-dimensional geometry in optically transparent media. The accuracy and computational efficiency of the VF approach greatly exceeds that of the raytracing method (RTM).

In the case of absorbing media, the accurate treatment of attenuation and VF in the vicinity of the surface is performed. The absorption of radiation brings significant computational expenses in the radiation transfer model. However, such computational cost is related mostly to the optical model and, to a lesser degree, to the VF approach itself. Moreover, the calculation of radiation flux under the assumption of optically transparent media by means of the VF approach provides an efficient and accurate estimation of the flux density incoming from the shock wave.

The advantage of radiation transfer model based on the original gasdynamic grid rather than on the grid in a local system of coordinates is demonstrated. The overall efficiency of the VF approach can be two orders of magnitude higher than the RTM. At the same time, the cost of the VF approach demonstrates less sensitivity to the grid refinement than that of the RTM.

\section{References}

[1] Sutton, K., "Air Radiation Revisited," Proceeding of AIAA 19th Thermophysics Conference, AIAA Paper 1984-1733, 1984.

[2] Olynick, D., Chen, Y.-K., and Tauber, M. E., "Aerothermodynamics of the Stardust Sample Return Capsule," Journal of Spacecraft and Rockets, Vol. 52, No. 6, 1999, pp. 442-462. doi: $10.2514 / 2.3466$

[3] Desai, P. N., Lyons, D. T., Tooley, J., and Kangas, J., "Entry, Descent, and Landing Operations Analysis for the Stardust Entry Capsule," Journal of Spacecraft and Rockets, Vol. 45, No. 6, 2008, pp. 1262-1268. doi: $10.2514 / 1.37090$

[4] Whiting, E., Park, C., Liu, Y., Arnold, J., and Paterson, J., "NEQAIR 96 User Manual," NASA Tech. Rept. RP-1389, 1996.

[5] Glassner, A. S., An Introduction to Ray Tracing, Morgan Kaufmann, San Francisco, CA, 1989, pp. 1-32.

[6] Surzhikov, S. T., "Computing System for Solving Radiative Gasdynamic Problems of Entry and Re-Entry Space Vehicles," Radiation of High Temperature Gases in Atmospheric Entry, edited by Warmbein, B., Vol. 533, ESA Publ. Division, Noordwijk, The Netherlands, 2003, pp. 111-118.

[7] Mazaheri, A., Johnston, C. O., and Sefidbakht, S., "Three-Dimensional Radiation Ray-Tracing for Shock-Layer Radiative Heating Simulations," Journal of Spacecraft and Rockets, Vol. 50, No. 6, 2013, pp. 485-493. doi:10.2514/1.A32448

[8] MacCormack, R. W., "The Carbuncle CFD Problem," Proceeding of 49th AIAA Aerospace Sciences Meeting, AIAA Paper 2011-381, 2011.

- [9] Jeans, J., "The Equations of Radiative Transfer of Energy," Monthly Notices of the Royal Astronomical Society, Vol. 78, No. 1, 1917, pp. 28-36. doi:10.1093/mnras/78.1.28

[10] Andrienko, D. A., and Surzhikov, S. T., " $P^{1}$ Approximation Applied to the Radiative Heating of Descent Spacecraft," Journal of Spacecraft and Rockets, Vol. 49, No. 6, 2012, pp. 1088-1098. doi:10.2514/1.A32169

[11] Andrienko, D. A., Surzhikov, S. T., and Shang, J. S., "Spherical Harmonics Method Applied to the Multi-Dimensional Radiation Transfer," Computer Physics Communications, Vol. 184, No. 10, 2013, pp. 2287-2298. doi:10.1016/j.cpc.2013.05.015

[12] Hottel, H., and Cohen, E., "Radiant Heat Exchange in a Gas-Filled Enclosure: Allowance for Nonuniformity of Gas Temperature," AIChE Journal, Vol. 4, No. 1, 1958, pp. 3-14. doi:10.1002/(ISSN)1547-5905

[13] Modest, M. F., Radiative Heat Transfer, Academic Press, Oxford, 2003, pp. 129-159.

[14] Siegel, R., and Howell, J. R., Thermal Radiation Heat Transfer, Taylor and Francis, Boca Raton, FL, 2002, pp. 881-888.

[15] Olejniczak, J., Wright, M., Prabhu, D., Takashima, N., Hollis, B., Zoby, E. V., and Sutton, K., "An Analysis of the Radiative Heating Environment for Aerocapture at Titan," AIAA Paper 4953-2003, 2003, p. 250.

[16] Bose, D., and Wright, M. J., "View-Factor Based Radiation Transport in a Hypersonic Shock Layer," Journal of Thermophysics and Heat Transfer, Vol. 18, No. 4, 2004, pp. 553-555. doi: $10.2514 / 1.8817$

[17] Lockwood, M. K., “Titan Aerocapture Systems Analysis," AIAA Paper 4799-2003, 2003, pp. 20-23.

[18] Liu, Y., Prabhu, D., Trumble, K. A., Saunders, D., and Jenniskens, P., "Radiation Modeling for the Reentry of the Stardust Sample Return Capsule," Journal of Spacecraft and Rockets, Vol. 47, No. 5, 2010, pp. 741-752. doi: $10.2514 / 1.37813$

[19] Park, C., "Review of Chemical-Kinetic Problems of Future NASA Missions, I-Earth Entries," Journal of Thermophysics and Heat Transfer, Vol. 7, No. 6, 1993, pp. 385-398. doi: $10.2514 / 3.431$

[20] Shang, J., and Surzhikov, S., "Simulating Nonequlibrium Flow for Ablative Earth Reentry," Journal of Spacecraft and Rockets, Vol. 47, No. 5, 2010, pp. 806-815. doi: $10.2514 / 1.49923$

- [21] Shang, J., Andrienko, D., Huang, P., and Surzhikov, S., "A Computational Approach for Hypersonic Nonequilibrium Radiation Utilizing Space Partition Algorithm and Gauss Quadrature," Journal of Computational Physics, Vol. 266, No. 6, 2014, pp. 1-21. doi:10.1016/j.jcp.2014.02.007

[22] Grantham, W. L., "Flight Results of a 25000-Foot-Per-Second Reentry Experiment Using Microwave Reflectometers to Measure Plasma Electron Density and Standoff Distance," NASA TN-D-6062, 1970. 
[23] Candler, G. V., and MacCormack, R. W., "Computation of Weakly Ionized Hypersonic Flows in Thermochemical Nonequilibrium," Journal of Thermophysics and Heat Transfer, Vol. 5, No. 6, 1991, pp. $266-273$. doi: $10.2514 / 3.260$

[24] Surzhikov, S., and Shang, J., "Numerical Rebuilding of Fire II Flight Data with the Use of Different Physical Chemical Kinetics and Radiation Models," Proceeding of AIAA 51 st AIAA Aerospace Sciences Meeting Including the New Horizons Forum and Aerospace Exposition, AIAA Paper 2013-0190, 2006.

[25] Wright, M. J., Bose, D., and Olejniczak, J., "Impact of FlowfieldRadiation Coupling on Aeroheating for Titan Aerocapture," Journal of Thermophysics and Heat Transfer, Vol. 19, No. 1, 2005, pp. 17-27. doi:10.2514/1.10304

[26] Abramowitz, M., and Stegun, I. A., Handbook of Mathematical Function with Formulas, Graphs, and Mathematical Tables, Dover, New York, 1970, pp. 587-626.

[27] Surzhikov, S. T., Capitelli, M., Colonna, G., and Gorse, C., "Radiative Gas Dynamic Model of Hydrogen Laser-Supported Plasma Generator," Journal of Thermophysics and Heat Transfer, Vol. 22, No. 1, 2008, pp. 62-70.

doi: $10.2514 / 1.29982$

[28] Andrienko, D. A., "Non-Equilibrium Models for High Temperature Gas Flows," Ph.D. Thesis, Wright State Univ., Dayton, OH, 2014.

[29] Goulard, R., "The Coupling of Radiation and Convection in Detached Shock Layers," Journal of Quantitative Spectroscopy and Radiative Transfer, Vol. 1, No. 6, 1961, pp. 249-257. doi:10.1016/0022-4073(61)90025-5

[30] Surzhikov, S., "Three Dimensional Simulation of Shock Layer Ionization for Ram-c II Flight Tests," Proceeding of 52nd Aerospace Sciences Meeting, AIAA Paper 2014-1078, 2014.

[31] Walpot, L. M. G. F. M., "Development and Application of a Hypersonic Flow Solver,” Ph.D. Thesis, TU Delft, Delft Univ. of Technology, Delft, The Netherlands, 2002.

[32] Chandrasekhar, S., Radiative Transfer, Courier Dover, New York, 1960, pp. 11-12.

K. Edquist Associate Editor 